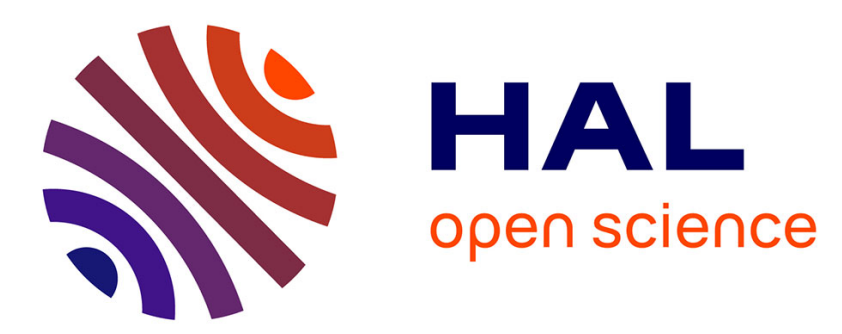

\title{
Reciprocal Kinematic Control: using human-robot dual adaptation to control upper limb assistive devices
}

Mathilde Legrand, Étienne de Montalivet, Florian Richer, Nathanaël Jarrassé, Guillaume Morel

\section{To cite this version:}

Mathilde Legrand, Étienne de Montalivet, Florian Richer, Nathanaël Jarrassé, Guillaume Morel. Reciprocal Kinematic Control: using human-robot dual adaptation to control upper limb assistive devices. Hamlyn Symposium on Medical Robotics, Jun 2019, Londres, United Kingdom. hal-02324425

\section{HAL Id: hal-02324425 \\ https://hal.science/hal-02324425}

Submitted on 21 Oct 2019

HAL is a multi-disciplinary open access archive for the deposit and dissemination of scientific research documents, whether they are published or not. The documents may come from teaching and research institutions in France or abroad, or from public or private research centers.
L'archive ouverte pluridisciplinaire HAL, est destinée au dépôt et à la diffusion de documents scientifiques de niveau recherche, publiés ou non, émanant des établissements d'enseignement et de recherche français ou étrangers, des laboratoires publics ou privés. 


\title{
Reciprocal Kinematic Control: using human-robot dual adaptation to control upper limb assistive devices
}

\author{
M. Legrand ${ }^{1}$, E. de Montalivet ${ }^{1}$, F. Richer ${ }^{1}$, N. Jarrassé 1 , G. Morel ${ }^{1}$ \\ 'Sorbonne University, CNRS, UMR7222 / INSERM, ISIR-Agathe, Paris, France \\ \{mathilde.legrand,nathanael.jarrasse,guillaume.morel\}@sorbonne-universite.fr
}

\section{INTRODUCTION}

Upper limb (UL) assistive robots, such as exoskeletons, prostheses or supernumerary limbs, can rarely be fully autonomous devices. Indeed, it is generally not possible to use pre-defined patterns of motions because of the great diversity of tasks and the variety of UL movement strategies to achieve any of them. Control has to be provided to the users. For that purpose, the most widespread solutions to obtain user's motor intention use physiological signals (electromyograms or electroencephalograms e.g.) [1,2], distal functional joints (for instance, head or foot motions control the endeffector position and/or orientation)[3,4] or inter-joint synergies models[5]. Despite interesting results, they all still have important limitations: the first two are neither natural nor intuitive and suffer from robustness issues, the third one does not allow very versatile devices.

To tackle these issues, we propose a new control approach, together with a new paradigm, that uses the motion strategies naturally developed by the Central Nervous System (CNS). When a limb mobility is reduced, or when an assistive device does not work properly, CNS compensates and takes advantage of motor redundancy of the body: it calls other joints to still perform the desired gesture. Typical compensatory joints for UL movements are the trunk and the scapula[6]. Our concept is to servo the robot to these body compensations. The only task of the latter is to make its user come back to a comfortable posture, and this indirectly leads to the realisation of the intended motion. The reciprocal adaptation between human and robot allows both to reduce the body compensations and perform UL movements with the assistive device. We validated a proof of concept of this paradigm on ten healthy subjects who executed a path-tracking task with an elbow exoskeleton.

\section{MATERIALS AND METHODS}

\section{Control law}

The control law is built in such a way that the user only has to focus on the end-effector (EE) position, as he/she would have done with a healthy arm. The aim is that the EE position, first reached with body compensations, remains constant, while the device moves and makes the user's compensatory joints go back to a reference position. An integration step is then added to prevent any rigid position-position coupling that does not enhance the mobility.

The approach has been first applied to control an elbow joint with the following law (see Fig.1(a) for the definition of the anatomical parameters):

1. Compute $\delta$ the distance between the EE position and the reference position of the acromion (chosen to define a non-compensatory posture, as it reflects both trunk and scapula, the main joints involved in upper body compensations),

2. Compute $\beta_{\mathrm{n}}$ the angle the elbow should have to allow the EE to be in its current position without any compensation, according to

$$
\beta_{n}=\Pi-\operatorname{acos}\left(\frac{L_{f a}^{2}+L_{u a}^{2}-\delta^{2}}{2 L_{u a} L_{f a}}\right)
$$

with $\mathrm{L}_{\mathrm{ua}}$ and $\mathrm{L}_{\mathrm{fa}}$ the lengths of the upper arm and the forearm respectively.

3. Compute the angular velocity command to be sent to the robotic elbow:

$$
\dot{\beta}=\lambda \Delta \beta=\lambda\left(\beta_{n}-\beta\right) \quad(\text { Eq. } 2)
$$

with $\beta$ the current elbow angle and $\lambda$ the gain of the integrator set to 2. An activation threshold $(\Delta \beta>5$ degrees) was set to avoid instabilities.

\section{$\underline{\text { Validation }}$}

(a)

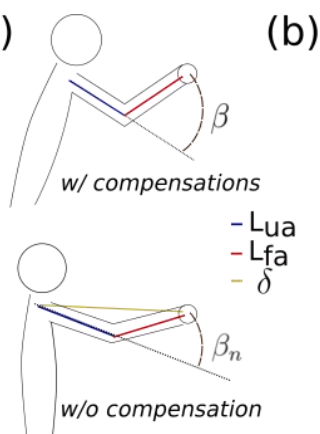

(b)

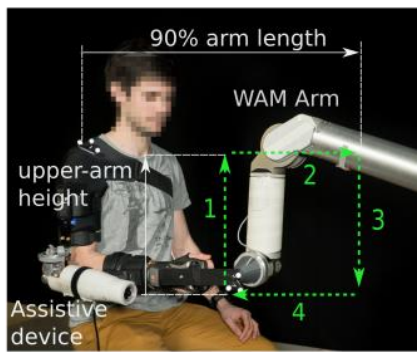

Fig.1(a)Anatomical parameters of the control law. (b)Experimental set-up. A WAM®Arm draws a rectangle in the sagittal plane of the subject; it pauses briefly at each corner. Numbers 1 to 4 indicate the movements steps. The dimensions are adapted to the subject's morphology. The subject is wearing a robotic joint acting like an elbow exoskeleton and a wrist splint with a rod attached to it.

Ten healthy subjects, aged 20-23, who all gave their written consent, wore an elbow exoskeleton prototype that guided their motions. The task consisted in following a moving target, carried by a WAM®Arm (Barrett Technology), that drew a rectangle in the sagittal plane of the subject. The dimensions of the rectangle were adapted to the subject's morphology (see Fig.1(b)). There were five repetitions. The task was performed in three different ways (later called modes): 
1. Natural $(\mathrm{N})$ : the subject did not wear the exoskeleton but moved freely, without any specific instructions nor constrains. This is to be used as the reference.

2. Elbow fixed $(\mathrm{F})$ : the robotic joint was locked at 90 degrees, it prevented any elbow motions. This worstcase scenario showed the body compensations that can be exhibited when the mobility is reduced.

3. Reciprocal Kinematic (RK) control: the subject wore the device which, commanded with the proposed control scheme, guided the movements of his/her elbow. The end-effector and the acromion positions were tracked in real time $(\mathrm{f}=100 \mathrm{~Hz})$ with the motion capture system Optitrack (NaturalPoint Inc.). The reference position was defined as the initial position of the subject. The subjects received no instructions nor explanation; they were only told to perform the task and that the assistive device would help them. A training on ten repetitions was allowed before recording five.

\section{RESULTS}

The first thing to notice is that the task was correctly performed with the three modes (no statistical difference between the precision errors). To evaluate the performance of RK control, several metrics were analysed; two are presented here: the range of motion (ROM) of the acromion, which represents the compensatory displacements, and the ROM of the elbow (see Fig. 2). Statistical analysis was performed: Lilliefors test was used to assess the normality of the data, then general linear models for normally distributed data and nonparametric Friedman test for the others.

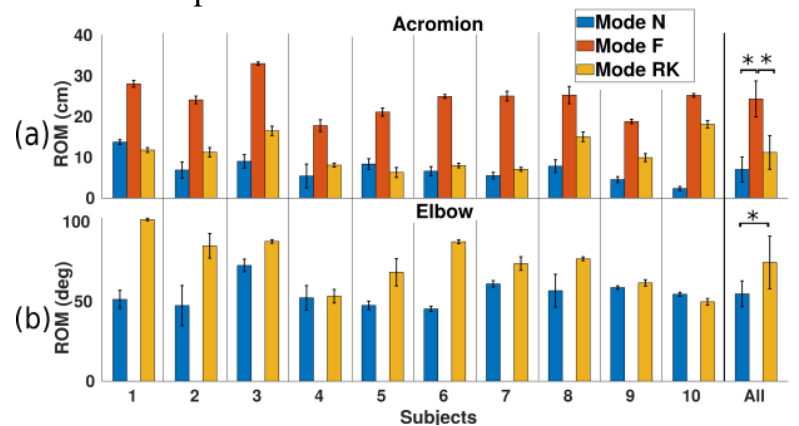

Fig.2 Performance of RK controlled-motions compared to natural and fixed-elbow ones. (a) ROM of the acromion. (b) ROM of the elbow. Metrics are averaged over the five trials and last columns is the mean over the ten subjects.

The ROM of the acromion is significantly higher for the F-mode than for $\mathrm{N}$ and $\mathrm{RK}$ ones $(\mathrm{p}<0.05)$ while no statistical difference exists between $\mathrm{N}$ - and RK-mode. This confirms that, due to reciprocal adaptation, RK control indeed reduces the user body compensations.

Concerning the elbow ROM, F-mode is absent as there is no movement of the joint. With RK-mode, we see an over-extension compared to the natural ROM. More detailed analysis shows that this over-extension appeared in the third stage of the movement, when the target is the furthest.

\section{DISCUSSION}

To build a natural and intuitive control for UL assistive devices, we propose to servo the robot to the body compensations of its user. This new paradigm, in which the device only focuses on correcting its user's posture, was tested with an elbow exoskeleton. It makes it possible to perform a path-tracking task, while requiring only minimal compensatory movements to work. Without any explanation provided, the users mastered the use of the device with RK in few trials, which highlights the intuitiveness of the control.

Some points of our experiment yet deserved more detailed comments. First, the motions performed with RK tend to show an over-extended elbow in the third stage of the task. This could be because the subjects leaned on one side to see better their end-effector, which led to unwanted elbow activations. This points out that the trunk is not always compensatory but can be functional (i.e. essential to do the task). We are working to detect the utilisation of the trunk (compensation or function) and adapt the answer of the device to avoid undesirable activations. Second, we worked with a motion capture system, which cannot be used in everyday life. However, the information it gave (positions of EE and acromion) can be obtained with other techniques, more suitable for home-use. For instance, it shall be possible to do the same with a simple set of two IMUs, one on the trunk and one on the arm, along with few anatomical data of the subject.

The preliminary results we obtained attest the usability and the intuitiveness of RK control. They support future tests on disabled subjects (amputees or post-stroke patients e.g.) as well as further developments for several degrees of freedom assistive devices. Moreover, RK control is task-independent, as the error vector used in the control law depends only on the reference posture (neither tasks nor compensations are explicitly defined). The experimental test of this versatility with different tasks is scheduled in the very near future. The reference posture was fixed for this experiment but further studies will make possible to have an automatically adjusted one. RK control is thus promising for a natural and versatile control of UL assistive devices, from prostheses to exoskeletons or even supernumerary arms.

\section{REFERENCES}

[1] Oskoei M, Huosheng H. Myoelectric control systems - A survey. Biomed Signal Proces 2007 July;2: 275-294.

[2] Velliste M, Perel S, Spalding M, Whitford A, Schwartz A. Cortical control of a prosthetic arm for self-feeding, Nature 2008; 453(7198):1098-1101.

[3] Resnik L, Klinger Sh, Etter K, Fantini C. Controlling a multi-degree of freedom upper limb prosthesis using foot controls: User experience. Disabil Rehabil Assist Technol 2014; 9(4):318-329.

[4] Jackowski A, Gebhard M, Thietje R. Head motion and head gesture based robot control: a usability study. IEEE TNSRE 2017 Oct;26(1): 161-170.

[5] Merad M, de Montalivet E, Roby-Brami A, Jarrassé N. Intuitive prosthetic control using upper limb inter-joint coordinations and IMU-based shoulder angles measurement: a pilot study, IROS 2016.

[6] Cirstea M, Levin M. Compensatory strategies for reaching in stroke. Brain 2000; 123;940-953. 